\title{
Analysis of "Spirituality" Category and its Structure in the English Language
}

\author{
Akhmedova Mehrinigor Bahodirovna, Baqoyeva Muhabbat Kayumovna
}

\begin{abstract}
Lexico-semantic category of "spirituality" is usually analyzed by the ways of defining concepts of the term. On the one hand, it seems to us that using these concepts simultaneously makes opposed ideas towards the meaning, however, the term " spirituality" is defined in different ways by different authors and it makes the concept complicated. The main point of the article is to analyze and categorize the term "spirituality" and give full answer to the readers about the English concept "spirituality".
\end{abstract}

Keywords : spirituality, religious level, faith, hope, peace, empowerment, lexica- semantic category, moral values, concept, human mind, definition.

\section{INTRODUCTION}

$\mathrm{I}_{\mathrm{n}}$ English, spirituality is expressed not only as a religious level but also as a larger concept as well. As R. Tanyi explained the term as following and it is necessary to mention that this definition is wider than the others:

"Spirituality is a personal search for meaning and purpose inlife, which may or may not be related to religion. It entails connection to self-chosen and/or religious beliefs, values, and practices that give meaning to life, thereby inspiring and motivating individuals to achieve their optimal being. This connection brings faith, hope, peace, and empowerment. The results are joy, forgiveness of oneself and others, awareness and acceptance of hardship and mortality, a heightened sense of physical and emotional well-being, and the ability to transcend beyond the infirmities of existence".[9;506-509]

As it is mentioned, spirituality is firstly connected with human's feeling of satisfaction from his own life. Thus, in contrast to the consciousness of the deductive thinking, i.e. the conviction that a solid faith can bring meaning to human life, a person's understanding of the essence of his or her life and his intentions to set goals for himself, as well as inductive thinking that relies on such a reality.

Revised Manuscript Received on July 22, 2019

Akhmedova Mehrinigor Bahodirovna A senior teacher of English literature department, Bukhara State University, Uzbekistan mexrishka82@mail.ru

Baqoyeva Muhabbat Kayumovna, A professor of English literature department, Bukhara State University, Uzbekistan

\section{METHODS AND ANALYSIS}

Thus, English spirituality takes into consideration both people's following certain moral values and one's own principles, beliefs. That's why in English spirituality has meaning for all people in the world as well as for each individual person. If we pay attention to the ideas which has been mentioned above, these moral values and principles arise some feelings such as belief, hope, and peacefulness. These feelings can be result of understanding happiness, forgiveness, transient of life. English people understand spirituality connected with happiness, peacefulness and hope concept spheres around the spirituality.

Differentiating these concepts in the human mind as abstract concepts, we see different shapes and scripts in every nation's thinking. That is, in the English mind, spirituality is not merely the result of religious and other beliefs, but in the common sense of the individual, and in the common sense of the norms of his own personal beliefs.

The distinctive features of the above differences play one of the key roles in shaping people's ideology. That is, the common goals of the peoples, their beliefs, their lifestyle and of course, the common goals that unite the nations as the people, and their common goals, as well as the nation or peoples, it is only expressed through the concepts of the same peoples. Understanding the past and future of a whole nation, by the same thing as the fact that only one concept is composed of different constituents in other people's mind. The concept of spirituality in Uzbek is not fully understood by English expression, and the concept of spirituality in English cannot be expressed in Uzbek alone. As the origin of these two elements and the way it differs from the public lifestyle, the conceptual structure of the script also consists of different concepts. Although the word representing a concept is different in different languages and cultures, the essence of their definition is the same as that of nations with different backgrounds, which is essentially the same as that of the concepts.

In English, spirituality is defined from religious outlook and it is explained like: "Spirituality can mean many things in popular usage, and is often understood differently by different people. While retaining a certain ambiguity, its current range of application extends from traditional institutional religion to occult practices. In general, the term appears to denote approaches to discovering, experiencing, and living out the implications of an authentic human life". $[7 ; 34]$

In "Oxford English Dictionary" the conception of the word "spirit" has twenty four 
meanings ${ }^{1}$. "The general meaning underlying all the uses is that of an animating or vital principle which gives life, transcending the material and mechanical. It refers to the essential nature of human beings, their strength of purpose, perception, mental powers, and frame of mind".[5;37]The spirit is accompanied by concepts such as the intended force, the perception, the power of consciousness, and the birth of consciousness, which are the prerequisite for the existence of a human being. However, it is difficult to regard the invasion as a peculiar reality. Because, it is a universal reality or events, such as perception of faith and aspiration for perfection

\section{DISCUSSION}

In scientific research on the concept of spirituality, this is characterized by a number of qualifiers. Especially, one of the characteristic features of spirituality is its inborn conception in human consciousness: "Human spirituality in a very real sense...unifies the whole person" and is "an inbuilt feature of the human species that develops from the beginning of an individual's life (or not) depending on conditions".[4;44,59] This complex concept has several categories in English language. Especially, it covers:

Category of "self-development";

"emotional" category;

category of "enlightenment";

category of "responsibility";

category of "consciousness";

category of "faith";

category of "aim";

category of "development";

category of "ethics".

Although, the concept of spirituality has been derived from religious beliefs, in the era of globalization this concept is gradually improving its meaning. This view is based on the following idea:"If we can accept concepts such as self-worth, self-esteem, and self-actualization, then it should be legitimate to explore...spirituality, for these concepts are equally as intangible as spirituality".[6; 16-19]We can conclude that the concept of spirituality can also be characterized as we feel the concept of self-worth, self-esteem, self-expression, as the concept is as above abstract. Bur the issue is not that of the notion of this concept, but of the fact that it can mean different values for each individual. This concept, along with a general explanation of the concept of spirituality, can be interpreted as personal improvement category, as it has a special meaning for each individual. The "emotional" category of spirituality is clearly expressed in the following description: "The spirit is dynamic. It must be felt before it can be conceptualized" This category can be regarded as a category of action, while being an emotional category: "In keeping with this view, terms like „spiritual growth" and ,development” are used to express the vibrant nature of spirituality'[8;7,119]

Every concept that possesses personal characteristics has sensational categories as well. If we take into consideration

the fact that spirituality means people's personal development, this concept comprises both positive and negative feelings that appear during the development period. Furthermore, people's development include person's background knowledge that is considered to be spiritual nutrition of human is also an important part of spirituality. Even though spirituality consists of different elements in the minds of human kind and nationalities, comprises different categories, this concept is built in some positive and normative ideas. Feelings like responsibility and conscience are basic tendencies of spirituality regardless of people's belief, culture or social status. Addition to this, these general categories are fundamental points to provide proportionality of the concept of spirituality in several languages. Concept to spheres of ideas that mean the same meaning can differ in semantic categories, but there are some categories that can be basic to consider them equivalent of the idea in two languages. Concepts like "conscience" or "responsibility" as a category of the idea of spirituality in the English language are main categories that can bind it with other concepts in other languages. According to connotative meaning of spirituality in English shown above it can be elucidated like this: "Spirituality is concerned with person's awareness of the existence and experience of inner feelings and beliefs, which give purpose, meaning and value to life. Spirituality helps individuals to live at peace with themselves, to love (God and) their neighbor and to live in harmony with the environment. For some, spirituality involves an encounter with God, or transcendent reality, which can occur in or out of the context of organized religion, whereas for others, it involves no experience or belief in the supernatural" $[3 ; 107]$

It can be seen that spirituality is not only personal or convictional concept but also socially important thing. National development is bounded with its thought progress. These alternations and progress can be seen in language as well. However, as a conversational means of humankind living in different areas English language shows this development more. It is clear that different nationalities members' personal development include different stages according to their residential area, national and cultural history, in other words, this procedure is apparent in various way for different nation's consciousness.

The concept of "spirituality" in English covers a number of concepts that serve to express the same meaning, or are components of the same complex meaning. We can add these notions as enlightenment, ethics, tolerance, patience, inspiration, development, self-development- personality improvement, consciousness, integrity, responsibility, belief, intelligence, fairness, courtesy, respect, enthusiasm, humanity, sense, skillfulness, dignity , friendship , patriotism, wisdom, knowledge, intellect, behavior, generosity, motivation , discernment, modesty, reticence, benevolence, shame, shamefacedness, decency.

Of course, since such concepts as the above represent an abstract, it is natural that variations in their description meet, or rather, complementary definitions. After all, such concepts have merged and created the perfect human portrait, each of

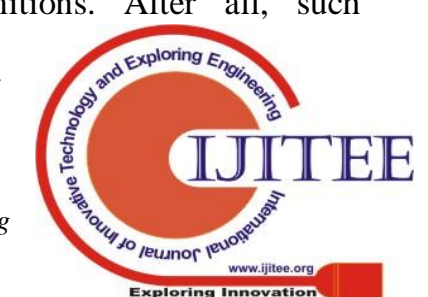


which has separate categories, can also be evaluated as a concept packed into a script and a frame. However, they are all indispensable, necessary part of a large-scale reality, such as"spirituality".

Most of the words that enter the system of"spirituality" can go to another grammatical category with the help of word-forming suffixes and prefixes, or go through other categories with the help of these tools to their current system. For example, the term enlightenment came from the verb enlighten - enlightening, the word wisdom and the horse wise came from the adjective. Hence, in English it can be noted that the units make up the concept of "spirituality" are vividly visualized by grammatical polyfunctionality.

Having touched on the direct analysis of these words, we must say that in English "spirituality" can not fully reveal the essence of reality, although the etymology of the origin of units that make up the category of nominative units has been regulated for some time by etymologically the scope of the subject. Nevertheless, we are far from denying the method of etymological grouping of words that make up the nominative units of "spirituality" in English, rather, the research in this direction has a tremendous linguistic significance. After all, linguistic-etymological study of the concept opens up a wide way to a comprehensive study of it in historical, cultural, psychological and sociological terms. Nevertheless, the study of these nominative units in the case of meaningful groups will be of great importance in finding the alternative of each concept in another language. Since, as it is known to all, these units, which are most often found in religious works, remain artistic, educational, in most cases, only one ethnic group is accepted as elegance realia. Of course, in this case, the etymological approach to these realities will not be able to fully express it all the same, although some point of reference to the issue.

The terms concerning the human psyche are formed only in the consciousness of One Nation and can not be realia, which is fulfilled in the speech of that nation. The reason is that the psyche does not form in the environment and does not develop, it expresses the same homogeneous human senses, feelings. So these universal senses, formed on the ground of feelings that are inherent in all flesh, this concept is only perceived as a nation-specific realia, is a somewhat misleading hypothesis. Consequently, the real sense of belonging to these concepts is not sentimental or nocturnal, but influenced by them, and gives insight into the nation that gave the name to the term.

In this regard, it is worthwhile to analyze the concept of "spirituality" in English, which is the main means of communication for a number of peoples as follows:

First of all, it is necessary to accept the concept of"spirituality" as a three-pointed reality. Thus, this desire has a mental, emotional and moral character.

The type of" Mental "of the nominative category"spirituality "can be attributed to the words of the group of meanings" mentality". An example of this is enthusiasm, wisdom, knowledge, intellect, development, self- development. The units in this group indicate the personal character trait of the concept of "spirituality".
As the second characteristic concept of "spirituality" it is possible to obtain terms that denote "emotional sense". The secret of such units includes inspiration, humanity, benevolence, shame, shamefacedness. While these concepts are related to emotional sense, the concept of"spirituality"also reveals the credential character of the concept.

And, finally, the third characteristic category of the concept of"spirituality" is the semantic units of "possession of moral character". In this category it is possible to make examples of ethics, tolerance, behavior, generosity, modesty. Moral terms determine the place of spirituality in society because of its social character.

\section{RESULTS AND CONCLUSION}

Proceeding from the above points, it is possible to divide the category of "spirituality" into three large groups. These are units that represent phenomena of consciousness, feelings and morality. Units related to the category of "consciousness" reflect to the understanding of the concept of "spirituality" in human thinking, units of "Nature" reveal the credential character of this concept.

Despite the fact that its components are three, the number of units in each group can be different in different languages. For this reason, in the minds of some nation, when spirituality becomes more of a contemplative concept, it is for another nation that this desire is an emotional category, again it means moral standards for representatives of another language. In the minds of representatives of different languages, one of these three categories prevails, as a result, the concept of "spirituality" is perceived through exactly the same dominant character units in the minds of the language representatives.

\section{REFERENCES}

1. Brown L. Oxford English Dictionary; Clarendon Press: Oxford, UK 1993. - P.

2. Chapman, L. Developing a useful perspective on spiritual health Wellbeing, spiritual potential and the search for meaning. Am. J. Health Prom. 1987 - P.39 - P.1

3. Fisher, J.W. Spiritual health: Its nature and place in the schoo curriculum. PhD thesis, University of Melbourne, 1998, Melbourne, Australia, $2010 \quad$ Available from http://eprints.unimelb.edu.au/archive/00002994/ \& Melbourne University Custom Book Centre: Melbourne, Australia, 2010 - P.107

4. Hay D.; Reich, K.H.; Utsch, M. Spiritual development: Intersections and divergence with religious development. In The Handbook of Spiritual Development in Childhood and Adolescence Roehlkepartain, E.C., King, P.E., Wagener, L.M., Benson, P.L., Eds. Sage Publications: Thousand Oaks, CA, USA, 2006 - P.59. - P.44.

5. Hill V. Spiritual development in the Education Reform Act: A source of acrimony, apathy or accord. Brit. J. Educ. Stud.1989, - P. 182. - P. 37.

6. Jose, N.; Taylor, E. Spiritual health: A look at barriers to its inclusion in the health education curriculum. Eta Sigma Gamman 1986, - P.18, 16-19

7. Muldoon M.; King, N. Spirituality, health care, and bioethics. - J. Relig. Health, 1995, - P 34

8. Priestley, J.G. Towards finding the hidden curriculum: A consideration of the spiritual dimension of experience in curriculum planning. Brit. J. Relig. Educ. 1985, - P. $119-$ P. 7.

9. Tanyi R. Nursing theory and concept development or analysis. Towards clarification of the meaning of spirituality. - Blackwell Science Ltd, Journal of Advanced Nursing, EBSCO Publishing, 2002. - P 509. - P. 506 